\title{
Control of complex quantum structures in droplet epitaxy
}

\author{
J. M. A. Chawner ${ }^{1}$, Y. Chang ${ }^{1}$, P. D. Hodgson ${ }^{1}$, M. \\ Hayne $^{1}$, A. J. Robson ${ }^{1}$, A. M. Sanchez ${ }^{2}$, Q. Zhuang ${ }^{1}$ \\ ${ }^{1}$ Department of Physics, Lancaster University, Lancaster, LA1 4YB, UK. \\ ${ }^{2}$ Department of Physics, Warwick University, Coventry, CV4 7AL, UK. \\ E-mail: j.chawner@lancaster.ac.uk
}

June 2019

\begin{abstract}
We report the controllable growth of GaAs quantum complexes in droplet molecular-beam epitaxy, and the optical properties of self-assembled $\mathrm{Al}_{x} \mathrm{Ga}_{1-x} \mathrm{As}$ quantum rings embedded in a superlattice. We found that $\mathrm{Ga}$ droplets on a GaAs substrate can retain their geometry up to a maximum temperature of $490^{\circ} \mathrm{C}$ during post-growth annealing, with an optimal temperature of $320^{\circ} \mathrm{C}$ for creating uniform and symmetric droplets. Through controlling only the crystallisation temperature under $\mathrm{As}_{4}$ in the range of $450^{\circ} \mathrm{C}$ to $580^{\circ} \mathrm{C}$, we can reliably control diffusion, adsorption and etching rates to produce various $\mathrm{GaAs}$ quantum complexes such as quantum dots, dot pairs and nanoholes. $\mathrm{Al}_{x} \mathrm{Ga}_{1-x} \mathrm{As}$ quantum rings are also realised within these temperatures via the adjustment of As beam equivalent pressure. We found that crystallisation using $\mathrm{As}_{2}$ molecules in the place of $\mathrm{As}_{4}$ creates smaller diameter quantum rings at higher density. The photoluminescence of $\mathrm{As}_{2}$ grown $\mathrm{Al}_{x} \mathrm{Ga}_{1-x} \mathrm{As}$ quantum rings embedded in a superlattice shows a dominant emission from the quantum rings at elevated temperatures. This observation reveals the properties of the quantum ring carrier confinement and their potential application as efficient photon emitters.
\end{abstract}

Keywords: Droplet Epitaxy, Molecular Beam Epitaxy, Quantum Structure, Photoluminescence, Atomic Force Microscopy, Transmission Electron Microscopy. 


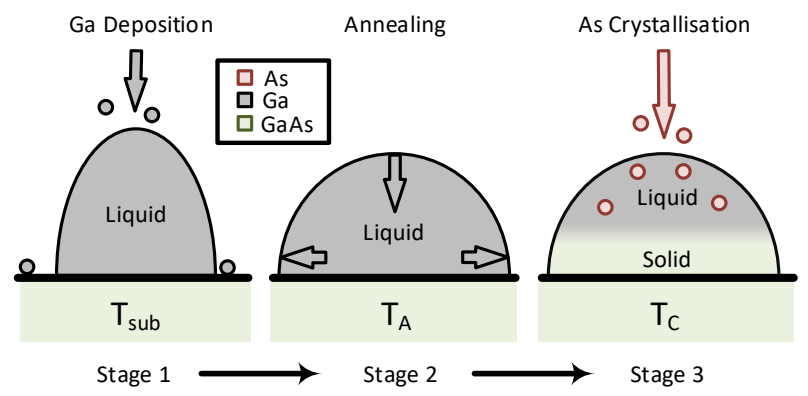

Figure 1. The three stages of droplet epitaxy growth. Stage 1: A few atomic monolayers of a group III element (e.g. Ga) is deposited. These nucleate into droplets, balancing the surface free energy at the droplet/vaccuum/surface interface. Stage 2: The substrate is heated to anneal the droplets. This affects the droplets dimensions due to a change in contact angle. Stage 3: A group $\mathrm{V}$ element (e.g. As) is deposited. This combines with the Ga rich droplets at the liquid/solid interface to create crystallised GaAs. This is done until the liquid material is entirely crystallised.

\section{Introduction}

Quantum dots (QDs) are nano-scale, zero-dimensional structures that confine charge carriers into quantised energy levels; a property which has extensive applications within optoelectronics and quantum computing $[1,2,3]$. A discrete energy system is observed within an atom's density of states, but whereas atoms have energy levels fixed by nature, the energy structure of QDs are directly related to their shape, material composition and strain. A successful approach for the fabrication of QDs is self-assembled growth by molecular beam epitaxy (MBE) or metalorganic chemical vapour deposition using the StranskiKrastanov technique (S-K), a layer-plus-island growth mode generated from lattice strain [4]. In S-K growth, particular material combinations need to be used, with a lattice mismatch of a few percent, limiting the options available. Dislocations can occur as a consequence of lattice strain [5].

One alternative to $\mathrm{S}-\mathrm{K}$ growth is droplet epitaxy (DE), which creates QDs from consecutive depositions of group III and V materials (Figure 1). DE can be a strain-free growth method, which helps reduce the likelihood and severity of defects and is therefore advantageous for optoelectronic devices [6]. In addition, the growth mechanism can be performed with a wider range of material combinations and substrate orientations than S-K growth, including those that are lattice-matched as well as lattice-mismatched [7]. Due to the consecutive nature of DE, each stage of epitaxy can be adjusted as desired. For example, it has been shown that the substrate annealing temperature at each stage of DE affects the resulting nanostructure architecture [8], with many different types of structures realised via $\mathrm{DE}[9,10,11,12]$, including nanowires [13]. This paper aims to explore the DE procedure to allow controlled and efficient production of specific quantum structures with high optical efficiency. For the device materials, GaAs and $\mathrm{Al}_{x} \mathrm{Ga}_{1-x} \mathrm{As}$ were chosen due to their type-I heterojunction properties, which are capable of fabricating optoelectronic singlephoton devices operating in the near-infrared band. Furthermore, the similarity in lattice constant between the two materials produces little to no strain, making GaAs $/ \mathrm{Al}_{x} \mathrm{Ga}_{1-x}$ As QDs inaccessible to the widely exploited S-K mode, and thus substantially less investigated than InAs/GaAs QDs, for example. QD's have been previously fabricated with these materials on a (111) surface to produce quantum entangled photons [14], a testament to the materials defect-free properties and optoelectronic abilities. They were also recently used to clearly demonstrate the existence of the somewhat elusive phonon bottleneck [15], while the lack of strain and considerable knowledge of the GaAs $/ \mathrm{Al}_{x} \mathrm{Ga}_{1-x}$ As system makes modelling of their properties relatively straight forward [16]. First, the effect of annealing temperature on metal Ga droplets is investigated on a GaAs substrate. Then we explore the effect of substrate temperature on the evolution of self-assembled GaAs nanostructures during As exposure for crystallisation. Various quantum complexes were obtained from the same recipe, with only the crystallisation temperature as a variable. A comparison of the effects of using molecules of $\mathrm{As}_{2}$ or $\mathrm{As}_{4}$ for crystallisation on an $\mathrm{Al}_{x} \mathrm{Ga}_{1-x} \mathrm{As}$ matrix was investigated. Using $\mathrm{As}_{2}$ simplifies the $\mathrm{MBE}$ growth procedure as no growth pause is needed to turn down the high cracker temperature, as would be the case for using $A s_{4}$. Finally, a sample of GaAs quantum rings (QRs) grown with optimal conditions and embedded in an AlAs/GaAs superlattice (SL), was fabricated. Photoluminescence (PL) studies of this sample reveal good zero-dimensional carrier confinement, indicating its potential application as a single photon emitter.

\section{Experimental Details}

The samples were grown in a conventional MBE reactor equipped with effusion cells for group III elements and a valved cracker for $\mathrm{As}_{4}$ or $\mathrm{As}_{2}$ molecules. A GaAs (001) substrate was heated to $580{ }^{\circ} \mathrm{C}$ and a $200 \mathrm{~nm}$-thick GaAs buffer layer was grown. Then the substrate temperature was reduced to $250^{\circ} \mathrm{C}$ with the As cracker temperature set to $650{ }^{\circ} \mathrm{C}$. The valve and shutter were closed for 30 minutes to optimise the background vacuum environment to $\approx 10^{-10}$ torr. Next, the droplet growth was performed by depositing 3 monolayers (MLs) of $\mathrm{Ga}$ at a growth rate of 


\begin{tabular}{|c|c|c|c|c|c|c|c|c|}
\hline Sample & Growth Surface & $T_{A}$ & $T_{C}$ & BEP & $h(\mathrm{~nm})$ & $d(\mathrm{~nm})$ & Density $\left(\mathrm{cm}^{-2}\right)$ & Structure \\
\hline $\mathrm{A}$ & $\mathrm{GaAs}$ & 320 & - & - & $8.0 \pm 0.2$ & $80 \pm 3$ & $(5.1 \pm 0.2) \times 10^{9}$ & Droplet \\
\hline B & GaAs & 490 & - & - & $5.5 \pm 0.5$ & $100 \pm 10$ & $(4.9 \pm 0.2) \times 10^{9}$ & Droplet \\
\hline $\mathrm{C}$ & GaAs & 580 & - & - & - & - & - & Lumpy \\
\hline $\mathrm{D}$ & GaAs & 320 & 450 & $10^{-5}$ & $8.3 \pm 0.5$ & $90 \pm 10$ & $(4.9 \pm 0.2) \times 10^{9}$ & $\mathrm{QD}$ \\
\hline $\mathrm{E}$ & GaAs & 320 & 520 & $10^{-5}$ & $6 \pm 1$ & $120 \pm 10$ & $(5.0 \pm 0.2) \times 10^{9}$ & QD pair \\
\hline $\mathrm{F}$ & GaAs & 320 & 580 & $10^{-5}$ & $-9.0 \pm 0.5$ & $100 \pm 10$ & $(5.2 \pm 0.2) \times 10^{9}$ & Nanohole \\
\hline G & $\mathrm{Al}_{0.32} \mathrm{Ga}_{0.68} \mathrm{As}$ & 320 & 520 & $10^{-6}$ & $1.5 \pm 0.5$ & $40 \pm 10$ & $(1.9 \pm 0.1) \times 10^{10}$ & QR \\
\hline $\mathrm{H}$ & $\mathrm{Al}_{0.32} \mathrm{Ga}_{0.68} \mathrm{As}$ & 320 & 520 & $10^{-6}$ & $0.9 \pm 0.1$ & $35 \pm 10$ & $(3.7 \pm 0.1) \times 10^{10}$ & $\mathrm{QR}\left(\mathrm{As}_{2}\right)$ \\
\hline I & GaAs & 320 & 520 & $10^{-6}$ & $10 \pm 1$ & $110 \pm 10$ & $(3.0 \pm 0.1) \times 10^{8}$ & $\mathrm{QR}\left(\mathrm{As}_{2}\right)$ \\
\hline
\end{tabular}

Table 1. A direct comparison of samples A-I studied here. $T_{A}$ and $T_{C}$ represent the substrate temperature during annealing and crystallisation respectively. BEP represents the beam equivalent pressure of As flux during the 10 min crystallisation. $h$ and $d$ represent the height and diameter of the final nanostructures respectively. Samples D-G were crystallised with As 4 , while samples $\mathrm{H}$ and I were crystallised with $\mathrm{As}_{2}$. All samples were created with $3 \mathrm{MLs}$ of Ga, except sample I which used $1 \mathrm{ML}$.

$0.5 \mathrm{ML} s^{-1}$ for 6 seconds at a substrate temperature of $250^{\circ} \mathrm{C}$ (Figure 1, stage 1). The sample was split ex situ into three separate segments and returned to the MBE vacuum storage. During this procedure the samples were stored under nitrogen for no more than 30 mins, to reduce oxidation. Each sample was then subjected to different annealing temperatures for $20 \mathrm{mins} ; 320^{\circ} \mathrm{C}$, $490^{\circ} \mathrm{C}$ and $580^{\circ} \mathrm{C}$ for samples $\mathrm{A}, \mathrm{B}$ and $\mathrm{C}$ respectively in Table 1. All annealing temperatures were ramped to at a rate between $10^{\circ} \mathrm{C} / \mathrm{min}$ and $40^{\circ} \mathrm{C} / \mathrm{min}$ to maintain a constant ramp time between samples.

A new batch of $\mathrm{Ga}$ droplets were annealed at $320^{\circ} \mathrm{C}$ (recipe of sample A) and were then split ex situ into 3 samples; $\mathrm{D}, \mathrm{E}, \mathrm{F}$, before being returned to the MBE chamber individually. The samples were subjected to 10 mins of $\mathrm{As}_{4}$ irradiation with a beam equivalent pressure (BEP) of $10^{-5}$ torr, each at a different substrate temperature throughout crystallisation, i.e, $450{ }^{\circ} \mathrm{C}, 520^{\circ} \mathrm{C}$ and $580^{\circ} \mathrm{C}$ for samples D, E and $\mathrm{F}$ respectively in Table 1. All crystallisation temperatures were ramped to at a rate between $10^{\circ} \mathrm{C} / \mathrm{min}$ and $40^{\circ} \mathrm{C} / \mathrm{min}$ to maintain a constant ramp time between samples. This was also done for all crystallisation temperature mentioned in future samples.

In order to investigate $\mathrm{As}_{2}$ as compared with $\mathrm{As}_{4}$ crystallisation, the recipe for sample $\mathrm{E}$ was again reproduced to create two new samples. However, before the Ga droplet deposition, a $50 \mathrm{~nm} \mathrm{Al}_{0.32} \mathrm{Ga}_{0.68} \mathrm{As}$ layer was grown on the GaAs (001) substrate to create a type-I nanostructure/surface heterojunction for optoelectronic functionality. The BEP was also reduced to $10^{-6}$ torr, which has been shown to encourage QR growth [17]. QR's were chosen for their good characteristics as photonic sources [18]. Each sample remained in the MBE chamber and was crystallised at $520^{\circ} \mathrm{C}$ with a $10^{-6}$ torr BEP, one under $\mathrm{As}_{4}$ and the other under $\mathrm{As}_{2}$ (samples $\mathrm{G}$ and $\mathrm{H}$ respectively in Table 1 ).

Finally, a sample of $\mathrm{As}_{2}$-crystallised QRs embed- ded in a SL (sample I in Table 1) was fabricated to study the optical properties of the QRs created by $\mathrm{As}_{2}$. Droplet growth was initiated on a GaAs (001) substrate with a $420 \mathrm{~nm}$ thick GaAs/AlAs $(2.8 \mathrm{~nm} / 2.8 \mathrm{~nm})$ SL structure. A 50-nm-thick $\mathrm{Al}_{0.32} \mathrm{Ga}_{0.68}$ As layer was then grown before QRs were fabricated with the an almost identical recipe to sample $\mathrm{H}$, but with a reduced liquid Ga volume of $1 \mathrm{ML}$, to limit the areal density of structures. This was originally done in an attempt to allow optical studies on a single QR, however the areal density was still too high to perform such a measurement, so the sample was tested as a whole. The GaAs QRs were capped by another 50-nm-thick layer of $\mathrm{Al}_{0.32} \mathrm{Ga}_{0.68} \mathrm{As}$, followed by another SL, to form a potential well to trap carriers locally in the QRs during optical stimulation. Then finally another set of QR's were fabricated on the surface for topographic observations. A cross section of a $\mathrm{QR}$ within sample I was observed via transmission electron microscopy (TEM). X-ray diffraction measurements of the sample were taken to analyse the structural details of the SL and the $\mathrm{Al} / \mathrm{Ga}$ balance. The optical properties were studied by PL measurements with a 532-nm laser for a temperature range of $4 \mathrm{~K}$ to $100 \mathrm{~K}$ in an Oxford Instruments flow cryostat. The morphology of all the quantum complexes was investigated with atomic force microscopy (AFM) on a Multimode 8 system.

\section{Results and Discussion}

In this discussion, surface processes during fabrication are approximately modelled to first order using the Arrhenius equation, which relates an event $x$ occurrence rate $R_{x}$ with surface location $\vec{r}$ and temperature $T$ [19]:

$R_{x}(\vec{r}, T)=v_{x} e^{-E_{x}(\vec{r}) / k_{B} T}$,

where $v_{x}$ typically represents an intrinsic frequency for the process, such as atomic vibration. $E_{x}$ is the 
activation energy for the event $x$ at position $\vec{r}$ and $k_{B}$ is the Boltzmann constant. This model has been used as the core principle in droplet epitaxy Monte Carlo simulations which have accurately recreated experimental observation [17, 20]. Each process rate is limited by intrinsic frequency $v_{x}$. When $k_{B} T \gtrsim \bar{E}_{x}$, the process rate becomes a significant fraction of the limiting frequency $v_{x}$, where $\bar{E}_{x}$ is the average activation energy over the surface defined by $\vec{r}$. Therefore, the processes may switch in dominance as temperature changes, dependent on $v_{x}$.

\subsection{Annealing effect on $G$ a droplets}

Figure 2 shows the AFM images of the Ga droplets annealed (Figure 1, stage 2) at temperatures of $320^{\circ} \mathrm{C}$, $490{ }^{\circ} \mathrm{C}$ and $580^{\circ} \mathrm{C}$ for samples $\mathrm{A}, \mathrm{B}$ and $\mathrm{C}$ respectively. It is evident that annealing has a significant effect on the geometry of the Ga droplets. Low annealing temperature (sample A) yielded droplets with a diameter of $80 \pm 3 \mathrm{~nm}$, height of $8.0 \pm 0.2 \mathrm{~nm}$ and an areal structure density of $(5.1 \pm 0.2) \times 10^{9} \mathrm{~cm}^{-2}$. A higher annealing temperature $490^{\circ} \mathrm{C}$ (sample B) led to droplets with a diameter of $100 \pm 10 \mathrm{~nm}$, height of $5.5 \pm 0.5 \mathrm{~nm}$ and a similar areal density of $(4.9 \pm 0.2) \times 10^{9} \mathrm{~cm}^{-2}$. Above this temperature a lumpy surface was produced without individual nanostructures (sample $\mathrm{C}$ at $580^{\circ} \mathrm{C}$ ). Assuming that the droplet/surface GaAs interface energy is lowered with higher temperature, the larger droplet diameter at $490^{\circ} \mathrm{C}$ (sample B) can be attributed to a reduced droplet contact angle and higher degree of surface wetting caused by the equilibrium of surface energy at the droplet/surface/vacuum interface [4]. Raising the temperature to $580^{\circ} \mathrm{C}$ (sample $\mathrm{C}$ ) demonstrates a more complete wetting of the surface, and therefore a loss of distinguishable nanostructures.

\subsection{Crystallisation under As exposure}

An investigation was made into the effect of substrate temperature during $\mathrm{As}_{4}$ crystallisation (Figure 1, stage 3 ) on final nanostructure geometry. The AFM scans in Figure 3 clearly show the capability of varying crystallisation temperature to introduce different forms of nanostructure, the dimensions of which are given in Table 1. Under As flux, many competing processes determine the movement and settling location of As adatoms, all of which can be modelled to first order via the Arrhenius equation (Equation 1). In the temperature range explored here, the main driving first order processes observed within the Ga droplets are diffusion, adsorption and etching of Ga and As liquid material. The intrinsic frequency for all the processes are dependent on many factors, including the Debye frequency of the substrate for surface processes and the surrounding density of $\mathrm{Ga}$ and As for all processes [20]. This creates variance in process rates between different adatoms.

At $450^{\circ} \mathrm{C}$ (sample D), single QDs were formed with a diameter of $90 \pm 10 \mathrm{~nm}$ and height of $8.3 \pm 0.5 \mathrm{~nm}$. This indicates that $\mathrm{Ga}$ and As surface adsorption rates $R_{A}$ are now active and dominant, allowing a complete and even crystallisation of the Ga droplet at the liquid/solid interface without diffusion or etching effects impacting the structure significantly.

Further increase of the temperature to $520^{\circ} \mathrm{C}$ (sample E) formed QD pairs with a total structure diameter of $120 \pm 10 \mathrm{~nm}$ in the [1]10] axis and height of $6 \pm 1 \mathrm{~nm}$. The orientation of the QD pairs demonstrate the anisotropic surface diffusion taking place. At this temperature, the As diffusion rate $R_{D}$ within the Ga droplet is more active, allowing the As adatoms to crystallise at the energetically favourable liquid/solid/vacuum triple point quicker than the liquid/solid interface. Without surface tension, the droplet collapses into itself, forcing material away from the centre. The potential energy surface of $\operatorname{GaAs}(001)$ attracts the liquid material anisotropically in the [1 $\overline{1} 0]$ and [110] directions $[21,22,23]$. This effect results in the crystallisation of two separate QDs that form opposite the original droplet centre.

At a substrate temperature of $580{ }^{\circ} \mathrm{C}$ (sample $\mathrm{F}$ ), the liquid material forms nanoholes within the GaAs substrate with a diameter of $100 \pm 10 \mathrm{~nm}$ and depth of $9.0 \pm 0.5 \mathrm{~nm}$. As observed previously [24], deposited $\mathrm{As}_{4}$ that comes into contact with the droplet diffuses outward from the centre and crystallises at the liquid/solid/vacuum interface faster than any other location (also observed in sample E). At this higher temperature, As dissolution into the Ga droplet is now fully activated and the etching rate $R_{E}$ has become the dominant process. The Ga droplet significantly etches the GaAs substrate at the liquid/solid interface, causing the liquid material to drill downwards. Liberated surface $\mathrm{Ga}$ and As supplement the liquid material and the As atoms diffuse through to crystallise at the liquid/solid/vacuum triple point.

These growth observations show that GaAs DE growth can create topographies with quantum artefacts at temperatures up to $580^{\circ} \mathrm{C}$ on an GaAs substrate. For the liquid material, $R_{A}$ has observable dominance up to $520^{\circ} \mathrm{C}, R_{D}$ has noticeable impact at $520^{\circ} \mathrm{C}$ and $R_{E}$ has dominance at $580^{\circ} \mathrm{C}$. The order of process dominance with increasing temperature, i.e. adsorption, diffusion, etching, agree with the activation energies for an As atom on the solid/liquid interface: $\bar{E}_{A}, \overline{E_{D}}, \overline{E_{E}}=0.5 \mathrm{eV}, 0.7 \mathrm{eV}, 0.9 \mathrm{eV}$ respectively [17]. However, the temperatures we report differ from previous findings which show dot-like growth occurs up to $250^{\circ} \mathrm{C}$ and ring-like growth occurs at around 

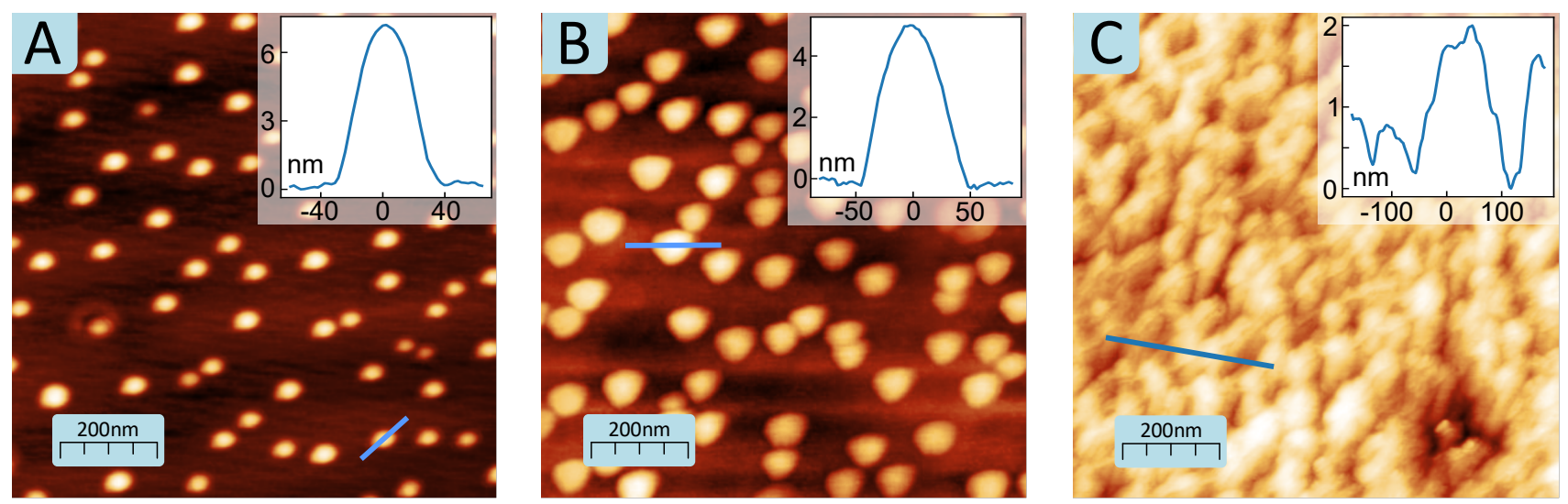

Figure 2. $1 \times 1 \mu \mathrm{m}^{2}$ AFM images and topographic profiles (insets) after 3 MLs of Ga deposition on a GaAs surface for the following substrate temperatures: sample A annealed at $320^{\circ} \mathrm{C}$, sample B at $490^{\circ} \mathrm{C}$ and sample $\mathrm{C}$ at $580^{\circ} \mathrm{C}$. All AFM images are topographic.
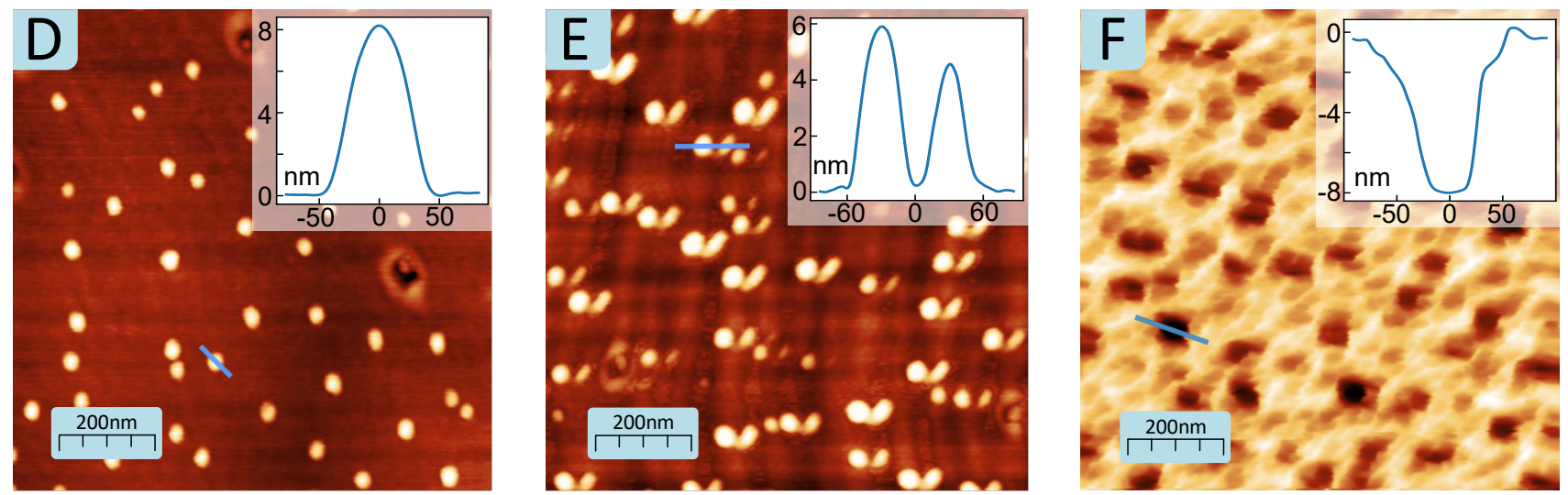

Figure 3. $1 \times 1 \mu \mathrm{m}^{2}$ AFM images and topographic profiles (insets) of crystallised samples after 10 mins of As 4 irradiation at BEP $10^{-5}$ torr at the following substrate temperatures: sample D at $450{ }^{\circ} \mathrm{C}$, sample $\mathrm{E}$ at $520^{\circ} \mathrm{C}$, sample $\mathrm{F}$ at $580^{\circ} \mathrm{C}$. All $\mathrm{AFM}$ images are topographic.

$350^{\circ} \mathrm{C}[17]$. This suggests that other fabrication parameters shifts the dominant processes during this type of growth, such as exposing our samples to the atmosphere during splitting, and differences in initial droplet dimensions. The samples $\mathrm{D}$ to $\mathrm{F}$ demonstrate the extensive range of nanostructures obtained by varying only the crystallisation temperature during growth (Figure 4).

\section{3. $A s_{2}$ and $A s_{4}$ Crystallisation Comparison}

The AFM images in Figure 5 show the quantum structure morphology of samples $\mathrm{G}$ and $\mathrm{H}$ crystallised at $520{ }^{\circ} \mathrm{C}$ with $\mathrm{As}_{4}$ and $\mathrm{As}_{2}$ respectively. Sample G contains QRs with diameter $40 \pm 10 \mathrm{~nm}$, height $1.5 \pm 0.5 \mathrm{~nm}$ and density $(1.9 \pm 0.1) \times 10^{10} \mathrm{~cm}^{-2}$, whereas sample $\mathrm{H}$ achieved QRs with diameter $35 \pm$ $5 \mathrm{~nm}$, height $0.9 \pm 0.1 \mathrm{~nm}$ and density $(3.7 \pm 0.1) \times$ $10^{10} \mathrm{~cm}^{-2}$. This shows a successful attempt to create QR's from a QD recipe with reduced BEP. By reducing the As BEP, liquid As becomes less available, reducing adsorption rate and allowing diffusion to have more impact on the growth. The $\mathrm{Al}_{0.32} \mathrm{Ga}_{0.68} \mathrm{As}$ surface does not encourage anisotropic diffusion. The nanorings developed following growth behaviour that has been observed in previous studies [25] and analysed theoretically $[26,17]$. We suggest the high areal structural density as compared to samples A-F can be attributed to both the liquid material reaction with the $\mathrm{Al}_{0.32} \mathrm{Ga}_{0.68} \mathrm{As}$ surface and the change in As BEP. The limited diameter and height of rings for sample $\mathrm{H}$ indicates that the $\mathrm{Ga}$ droplet has less time to diffuse at rate $R_{D}$ before crystallising, which agrees with previous studies showing more efficient incorporation of $\mathrm{As}_{2}$ than that of $\mathrm{As}_{4}$ [27, 28]. This is further evidenced by the reduced QR density observed in sample $\mathrm{G}$, as the $\mathrm{Ga}$ droplets have more time to nucleate together, due to a slower crystallisation and the growth pause required to tune the cracker temperature down for $\mathrm{As}_{4}$.

Using $\mathrm{As}_{2}$ in the place of $\mathrm{As}_{4}$ allows the fabrication of higher density structures with reduced diameters and height. This could be useful for higher 


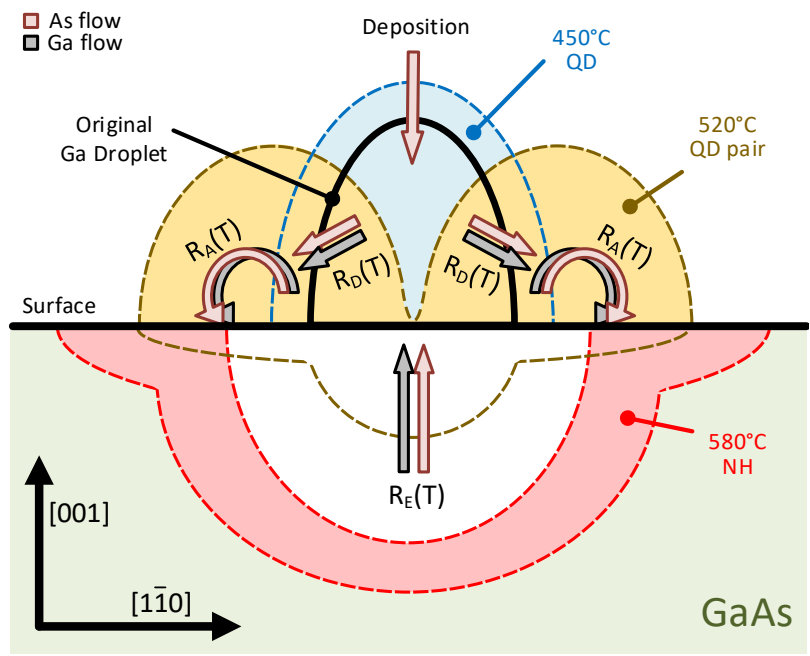

Figure 4. Schematic representation of various GaAs nanostructure topographical profiles formed experimentally from $\mathrm{Ga}$ droplets at various crystallisation temperatures (not to scale). Included are the evolution of Ga droplet into GaAs QDs at $450^{\circ} \mathrm{C}$ (sample D), GaAs QD pairs at $520^{\circ} \mathrm{C}$ (sample E) and GaAs nanoholes at $580^{\circ} \mathrm{C}$ (sample F). The internal structure of the nanoholes $(\mathrm{NH})$ has not been directly observed. $R_{D}, R_{E}$ and $R_{A}$ visualise the flow of diffusion, etching and adsorption rates respectively. $R_{D}$ increases with temperature, which reduces nanostructure height. $R_{A}$ is dominant at low temperature and allows crystallisation to occur before much diffusion takes place. $R_{E}$ increases with higher temperature allowing the Ga droplet to drill into the substrate further.
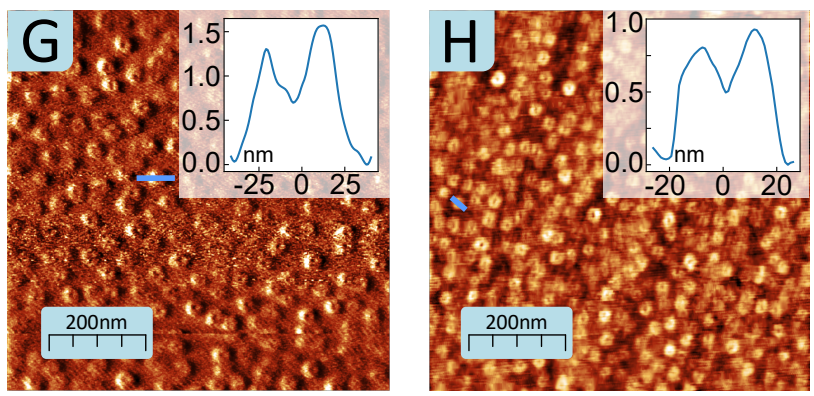

Figure 5. $1 \times 1 \mu \mathrm{m}^{2}$ AFM scans showing As 4 crystallised GaAs $/ \mathrm{Al}_{0.32} \mathrm{Ga}_{0.68} \mathrm{As}$ QRs (sample $\mathrm{G}$ ) and $\mathrm{As}_{2}$ crystallised $\mathrm{GaAs} / \mathrm{Al}_{0.32} \mathrm{Ga}_{0.68} \mathrm{As}$ QRs (sample $\mathrm{H}$ ). Both samples were crystallised at $520^{\circ} \mathrm{C}$ and BEP $5 \times 10^{-6}$ torr. For sample $\mathrm{G}$, a tapping-mode AFM phase image is used instead of a topographic scan to highlight the intricacy of the ring shape. Both the insets show the topographic profiles of a nanostructure from the respective scans.

energy electron/hole confinement and reduced emission wavelength for optical applications. In addition, there is no growth pause required to tune the $\mathrm{As}_{2}$ cracker temperature, which provides a more continuous growth procedure. The reduction of time between annealing and crystallisation limits Ga droplet nucleation and increases structure density, which can aid optical efficiency.
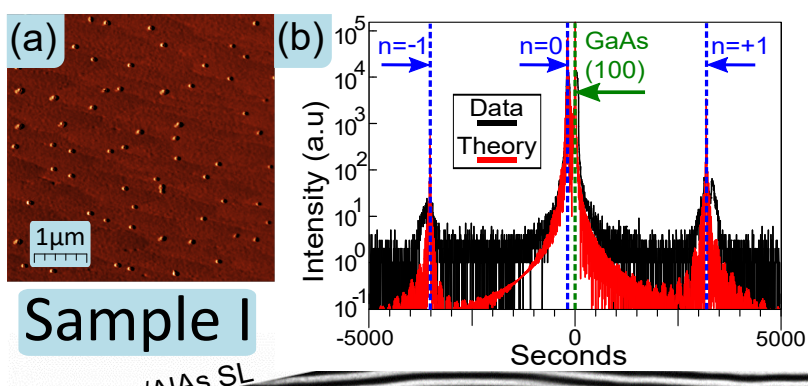

(c)
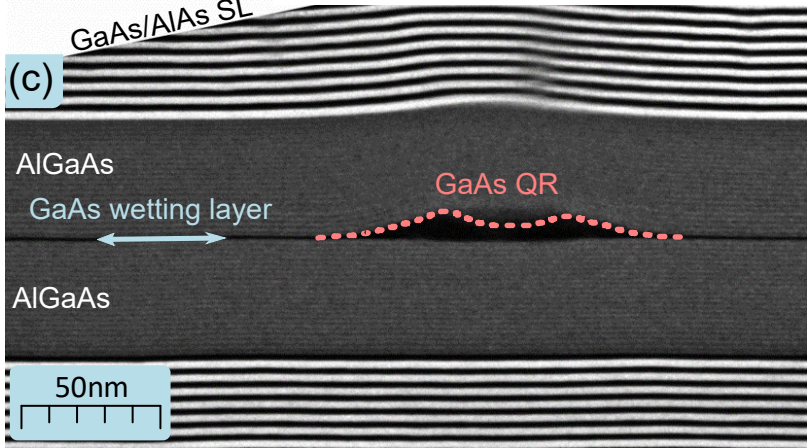

Figure 6. Measurements of sample I: (a) $5 \times 5 \mu \mathrm{m}^{2} \mathrm{AFM}$ scan showing As2 crystallised $\mathrm{GaAs} / \mathrm{Al}_{0.32} \mathrm{Ga}_{0.68} \mathrm{As}$ QRs (sample I). (b) X-ray diffraction of sample I centred on GaAs (100) reflection. The black line represents the measured intensity data, and the red line represents theoretical data generated from a model of the sample. (c) Cross-sectional bright-field TEM image of sample I showing the geometry of the SL structures, wetting layer and QR.

\subsection{Structural and Optical Properties of QRs Embedded in a Superlattice}

Figure 6(a) shows an AFM image of sample I's surface, the nanostructures have diameter $100 \pm 10 \mathrm{~nm}$, height $10 \pm 1 \mathrm{~nm}$ and density $(3.0 \pm 0.1) \times 10^{8} \mathrm{~cm}^{-2}$. Figure 6(b) shows X-ray diffraction data of sample I centred on GaAs (001) reflection, along with simulated results. To the left of the centre, the first Bragg angle from the SL can be observed (labelled $n=0$ ), the two peaks outward from the centre are the adjacent Bragg angles of the SL (labelled $n= \pm 1$ ), matching the theoretical model and suggesting a successful SL growth and correct $\mathrm{Al} / \mathrm{Ga}$ ratio. The bright-field TEM image in Figure 6(c) shows a cross section of sample I, partially cutting through a GaAs QR embedded between $\mathrm{Al}_{0.32} \mathrm{Ga}_{0.68} \mathrm{As}$ layers and the SL. The QR has a diameter of $\approx 90 \mathrm{~nm}$ and a height of $\approx 10 \mathrm{~nm}$. This is more than double the diameter of samples $\mathrm{G}$ and $\mathrm{H}$, suggesting that the extra fabrication steps to build the sandwich layers has interfered with nanostructure growth. It also shows the presence of a $2 \mathrm{~nm}$ thick GaAs wetting layer (thin quantum well) below the GaAs QRs. This has materialised from an As-rich $\mathrm{Al}_{0.32} \mathrm{Ga}_{0.68}$ As lower layer crystallising with the Ga deposited for creating droplets. The upper $\mathrm{Al}_{0.32} \mathrm{Ga}_{0.68} \mathrm{As}$ and $\mathrm{SL}$ have grown uniformly over the $\mathrm{QR}$, producing a curvature in these layers. 


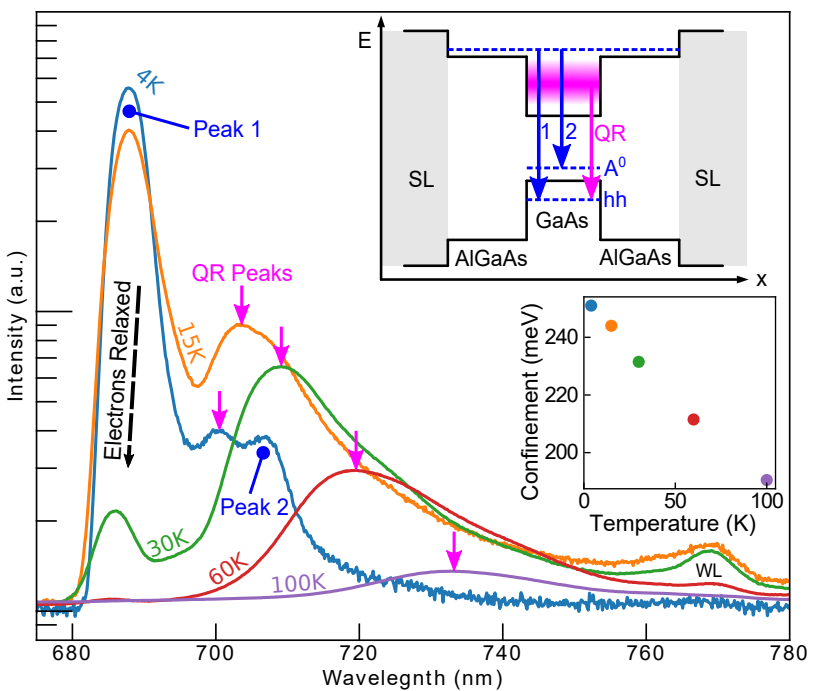

Figure 7. Log-scale photoluminescence (PL) intensity for sample I at a range of temperatures. The upper inset shows a schematic energy band diagram for the sample. At $4 \mathrm{~K}$ the $\mathrm{PL}$ is dominated by recombination from electrons in the $\mathrm{Al}_{0.32} \mathrm{Ga}_{0.68}$ As layer to heavy holes (hh) in the GaAs QR (Peak 1). Electrons also recombine with an occupied carbon impurity acceptor state $\left(\mathrm{A}^{0}\right)$ in the GaAs $\mathrm{QR}$ (Peak 2). QR emissions between $700 \mathrm{~nm}$ and $780 \mathrm{~nm}$ become dominant at temperatures of $30 \mathrm{~K}$ and above (QR Peaks), however, individual energy states can not be distinguished due to thermal broadening. The accumulative QR emission maxima (pink arrows) have been fitted with Varshni's empirical expression to show their modal confinement energy in the lower inset, i.e. the difference between the PL energy observed and the GaAs bandgap. As the temperature increases, electrons relax into lower $\mathrm{QR}$ states and the emission maximum shifts to higher wavelength. A weak wetting layer signal (WL) is observed at $769 \mathrm{~nm}$, across the temperature range.

Figure 7 shows PL measurements at temperatures from $4 \mathrm{~K}$ to $100 \mathrm{~K}$, which contain a range of emission peaks. At $4 \mathrm{~K}$, an intense peak at $689 \mathrm{~nm}$ (Peak 1) is observed from recombination of $\mathrm{Al}_{0.32} \mathrm{Ga}_{0.68} \mathrm{As}$ layer electrons to heavy holes (hh) in the QR valence band [15]. Another peak (Peak 2) can be distinguished at $706 \mathrm{~nm}$, which we attribute to recombination of electrons in the $\mathrm{Al}_{0.32} \mathrm{Ga}_{0.68}$ As with holes bound to a carbon impurity acceptor state $\left(\mathrm{A}^{0}\right)$ within the QR [15]. At $700 \mathrm{~nm}$ a signal is observed showing QR electron-hh recombination. At low temperature, electron access to pathways for relaxation is limited, suppressing transitions from GaAs QR states and causing peak 1 to be dominant. Peak 2 has low intensity, as it has been shown that it is associated with the presence of the phonon-bottleneck, which limits relaxation of electrons to $\mathrm{A}^{0}$ [15]. As the temperature is raised above $4 \mathrm{~K}$, an increase in available hh states lift the phonon bottleneck and suppress peak 2. More electrons can relax into the QR confined states, suppressing peak 1, and allowing a range of $\mathrm{QR}$ peaks between $700 \mathrm{~nm}$ and
$740 \mathrm{~nm}$ to become dominant. The various unresolved QR peaks show the accumulative signals from the confined states of the GaAs QRs. Higher temperature combined with inhomogeneous broadening makes each peak indistinguishable. Focusing on the accumulative luminescence maxima, a modal QR quantum confinement of $190-250 \mathrm{meV}$ at $100-4 \mathrm{~K}$ respectively is observed above the GaAs band gap as defined by Varshni's empirical expression [29]. The decreasing modal QR confinement with increasing temperature is caused by the lower confined states of the QRs becoming more easily accessed at higher temperature, when the carriers are thermalised and reach lower energy states before recombination [30]. PL emission from the wetting layer was observed at $768 \mathrm{~nm}$, although this signal is weak compared to the QR emission.

\section{Conclusion}

We have investigated the evolution and topography of self-assembled GaAs quantum structures created with droplet epitaxy growth. In the interest of producing uniform, defect free Ga droplets, the optimal postgrowth droplet annealing temperature was found to be $320^{\circ} \mathrm{C}$. During crystallisation under $\mathrm{As}_{4}$, the $\mathrm{Ga}$ and As surface atom diffusion, adsorption and etching processes can be controlled simply by adjusting the temperature of the sample. This single parameter can be chosen to create an extensive variety of complex GaAs nanostructures; quantum dots, quantum dot pairs and nanoholes. We find that surface adsorption is dominant at $450^{\circ} \mathrm{C}$, diffusion is activated at $520^{\circ} \mathrm{C}$, and etching is dominant at $580^{\circ} \mathrm{C}$. $\mathrm{As}_{2}$ reacts and crystallises liquid $\mathrm{Ga}$ at a higher rate than $\mathrm{As}_{4}$, and therefore is observed to produce smaller nanostructures at higher density. Also, $\mathrm{As}_{2}$ does not require a growth pause to adjust cracker temperature, therefore this material allows more efficient optical devices to be fabricated faster from the same materials. The realised low-density $\mathrm{As}_{2}$ quantum rings within a superlattice demonstrate strong zero-dimensional carrier confinement resulting in PL emission from several transitions between $700 \mathrm{~nm}$ and $750 \mathrm{~nm}$. These measurements imply that for droplet epitaxy, $\mathrm{As}_{2}$ is equally capable as $\mathrm{As}_{4}$ for creating high quality optoelectronic quantum structures and it removes the need to pause growth for tuning the cracker temperature. In particular, quantum rings in a superlattice created with $\mathrm{As}_{2}$ have good potential for use as efficient photon emitters.

[1] Kastner M A 1993 AIP Conference Proceedings 275 573586

[2] Ledentsov N, Ustinov V, Shchukin V, KopEv P, Alferov Z I and Bimberg D 1998 Semiconductors 32 343-365

[3] Lent C S and Tougaw P D 1997 Proceedings of the IEEE 85 541-557 ISSN 0018-9219 
[4] Lüth H 2001 Interfaces and Thin Films. Springer 4

[5] Smakman E, Garleff J, Young R, Hayne M, Rambabu P and Koenraad P 2012 Applied Physics Letters 100142116

[6] Wu J, Shao D, Dorogan V G, Li A Z, Li S, DeCuir E A, Manasreh M O, Wang Z M, Mazur Y I and Salamo G J 2010 Nano Letters 10 1512-1516

[7] DeJarld M, Reyes K, Smereka P and Millunchick J 2013 Applied Physics Letters 102133107

[8] Sablon K A, Lee J H, Wang Z M, Shultz J H and Salamo G J 2008 Applied Physics Letters 92203106

[9] Alonso-González P, Alén B, Fuster D, González Y, González L and Martínez-Pastor J 2007 Applied Physics Letters 91 163104

[10] Somaschini C, Bietti S, Koguchi N and Sanguinetti S 2009 Nano letters 9 3419-3424

[11] Somaschini C, Bietti S, Scaccabarozzi A, Grilli E and Sanguinetti S 2012 Crystal Growth \& Design 12 11801184

[12] Kunrugsa M, Panyakeow S and Ratanathammaphan S 2015 Journal of Crystal Growth 416 73-77

[13] Anyebe E, Zhuang Q, Sanchez A, Lawson S, Robson A, Ponomarenko L, Zhukov A and Kolosov O 2014 physica status solidi (RRL) - Rapid Research Letters 8658662 ISSN 1862-6254

[14] Kuroda T, Mano T, Ha N, Nakajima H, Kumano H, Urbaszek B, Jo M, Abbarchi M, Sakuma Y, Sakoda K, Suemune I, Marie X and Amand T 2013 Phys. Rev. B 88(4) 041306

[15] Chang Y C, Robson A, Harrison S, Zhuang Q and Hayne M 2015 AIP Advances 5067141

[16] Sidor Y, Partoens B, Peeters F M, Schildermans N, Hayne M, Moshchalkov V V, Rastelli A and Schmidt O G 2006 Physical Review B $\mathbf{7 3} 155334$

[17] Reyes K, Smereka P, Nothern D, Millunchick J M, Bietti S, Somaschini C, Sanguinetti S and Frigeri C 2013 Physical Review B $\mathbf{8 7} 165406$

[18] Fomin V M 2018 Quantum ring: A unique playground for the quantum-mechanical paradigm Physics of Quantum Rings (Springer) pp 3-32

[19] Vineyard G H 1957 Journal of Physics and Chemistry of Solids 3121 - 127 ISSN 0022-3697

[20] Vasilenko M A, Neizvestny I G and Shwartz N L 2015 Computational Materials Science 102 286-292

[21] Kley A, Ruggerone P and Scheffler M 1997 Physical review letters $\mathbf{7 9} 5278$

[22] Wang Z M, Holmes K, Mazur Y I, Ramsey K A and Salamo G J 2006 Nanoscale Research Letters 157 ISSN 1556$276 \mathrm{X}$

23] Adorno S, Bietti S and Sanguinetti S 2013 Journal of Crystal Growth $\mathbf{3 7 8} 515-518$

[24] Li X, Wu J, Wang Z M, Liang B, Lee J, Kim E S and Salamo G J 2014 Nanoscale 6 2675-2681

[25] Somaschini C, Bietti S, Sanguinetti S, Koguchi N and Fedorov A 2010 Nanotechnology 21125601

[26] Zhou Z Y, Zheng C X, Tang W X, Tersoff J and Jesson D E 2013 Phys. Rev. Lett. 111(3) 036102

[27] Garcia J, Neri C and Massies J 1989 Journal of Crystal Growth 98511 - 518 ISSN 0022-0248

[28] Holmes D, Belk J, Sudijono J, Neave J, Jones T and Joyce B 1995 Applied physics letters 67 2848-2850

[29] Varshni Y P 1967 physica 34 149-154

[30] Nuytten T, Hayne M, Bansal B, Liu H, Hopkinson M and Moshchalkov V V 2011 Physical Review B 84045302 